$\operatorname{IPPP} / 06 / 13$

$\mathrm{DCPT} / 06 / 26$

Cavendish-HEP-2006/07

\title{
MRST partons generated in a fixed-flavour scheme
}

\author{
A.D. MARTin ${ }^{a}$, W.J. Stirling ${ }^{a}$ And R.S. ThORnE ${ }^{c, 1}$ \\ ${ }^{a}$ Institute for Particle Physics Phenomenology, University of Durham, DH1 3LE, UK \\ ${ }^{c}$ Cavendish Laboratory, University of Cambridge, \\ JJ Thomson Avenue, Cambridge, CB3 0HE, UK
}

\begin{abstract}
We generate fixed three- and four-light-flavour sets of partons using MRST2004 partons as input. We show that it is important to set $n_{f}=3$ in the strong coupling, as well as in the splitting and coefficient functions, in order to obtain a consistent set of fixed-flavour partons. We compare the description of data using partons in both variableand fixed-flavour-number-schemes.
\end{abstract}

In deep inelastic scattering, and "hard" proton-proton (or antiproton-proton) high-energy collisions, the scattering proceeds via the partonic constituents of the hadron. To predict the rates of the various processes a set of universal parton distribution functions is required. These quark and gluon distributions are usually given in a variable-flavour-number-scheme (VFNS) in which the number of active quark flavours increases from $n_{f}=3$ to $n_{f}=4$, and then to $n_{f}=5$, as $Q^{2}$ increases above about $m_{c}^{2}$, and then above about $m_{b}^{2}$. Here $Q^{2}$ is the magnitude of the square of the momentum transfer in the process.

However there are practical reasons for also generating a set of up-to-date partons in the fixed three-light-flavour $\left(n_{f}=3\right)$ and fixed four-light-flavour $\left(n_{f}=4\right)$ schemes. For many exclusive or semi-inclusive processes the theoretical predictions for the hard scattering cross sections are, as yet, only available in the fixed-flavour-number-scheme (FFNS) where all the heavy flavours are produced in the hard cross-section. Thus fixed-flavour partons are needed in existing Monte Carlos, see, for example, $[1,2,3]$. Also, despite the availability of a number of general mass variable-flavour number schemes, extractions of partons from fits to structure function data are often performed using a FFNS, so it is useful to have available an up-to-date

\footnotetext{
${ }^{1}$ Royal Society University Research Fellow.
} 
parton set in order to make consistent comparisons and to judge the real differences between alternative parton sets obtained using the same theoretical framework.

Here we generate a set of FFNS partons by evolving up in $Q^{2}$ from a set of partons at the input scale, $Q_{0}^{2}=1 \mathrm{GeV}^{2}$, obtained in a VFNS global analysis of deep inelastic and related hard scattering data. The evolution differs from that used in the VFNS fit to the data in that

- we never turn on the heavy flavour $(c, b)$ contributions,

- we set $n_{f}=3$ in the splitting and coefficient functions,

- we keep $n_{f}=3$ in the coupling $\alpha_{s}\left(Q^{2}\right)$.

With respect to the last point, we usually define the coupling via the $n_{f}=4$ definition of $\Lambda_{\mathrm{QCD}}$; for example for the ( $\overline{\mathrm{MS}}, \mathrm{NLO}) \mathrm{MRST} 2004$ set of partons [4] we have $\Lambda_{\mathrm{QCD}}^{\left(n_{f}=4\right)}=347 \mathrm{MeV}$. We can convert this to a $n_{f}=3$ definition of $\Lambda_{\mathrm{QCD}}$ using the NLO relation [5]

$$
\Lambda^{(3)}=\Lambda^{(4)}\left(\frac{m_{c}}{\Lambda^{(4)}}\right)^{\frac{2}{27}}\left[2 \ln \left(\frac{m_{c}}{\Lambda^{(4)}}\right)\right]^{\frac{107}{2025}} .
$$

This relationship guarantees that the two couplings $\alpha_{S}$ are identical below $m_{c}^{2}$. However the three-flavour coupling runs more quickly above the charm quark transition point, and hence becomes smaller. The discrepancy between the speed of evolution increases further at the bottom transition point, and the three-flavour coupling has a value $\alpha_{S}^{(3)}\left(M_{Z}^{2}\right)=0.107$ compared to the value $\alpha_{S}^{(5)}\left(M_{Z}^{2}\right)=0.120$ obtained in the VFNS fit [4]. A similar behaviour is found for the CTEQ5 FFNS partons [6], where, there, the three-flavour coupling has a value $\alpha_{S}^{(3)}\left(M_{Z}^{2}\right)=0.106$ compared to the value $\alpha_{S}^{(5)}\left(M_{Z}^{2}\right)=0.118$. Thus, at high $Q^{2}, \alpha_{S}^{(3)}$ and $\alpha_{S}^{(5)}$ are significantly different quantities. Forcing $\alpha_{S}^{(3)}$ to take the value $\alpha_{S}^{(3)}\left(M_{Z}^{2}\right)=0.120$ would result in an enhanced coupling for $Q^{2} \sim 10 \mathrm{GeV}^{2}$, which would be inappropriate for describing data at these scales.

We emphasise that it is important to do the FF evolution consistently. We note that the determination of FFNS partons is very frequently done incorrectly, with a "variable-flavour" strong coupling, $\alpha_{S}$, used along with coefficient and splitting functions renormalized in a $n_{f}=3$ FFNS. Indeed this inconsistency between the renormalization scheme used for the coefficient functions and for the coupling was apparent in the original calculation [7] of the FFNS coefficient functions for deep inelastic scattering. The authors ${ }^{2}$ were fully aware of this discrepancy, but used both a VFNS coupling and VFNS partons since only VFNS partons were then available and because the error induced was small compared with other uncertainties. It is no longer the case that the error made from the wrong choice of coupling is so unimportant.

An illustration of the effect of such an inconsistency on a physical quantity is provided by the evolution of $F_{L}$. At leading order the gluon contribution to $F_{L}$ is

$$
F_{L}=\alpha_{S} C_{L g}^{1} \otimes g,
$$

\footnotetext{
${ }^{2}$ We thank Eric Laenen for clarifying this point.
} 

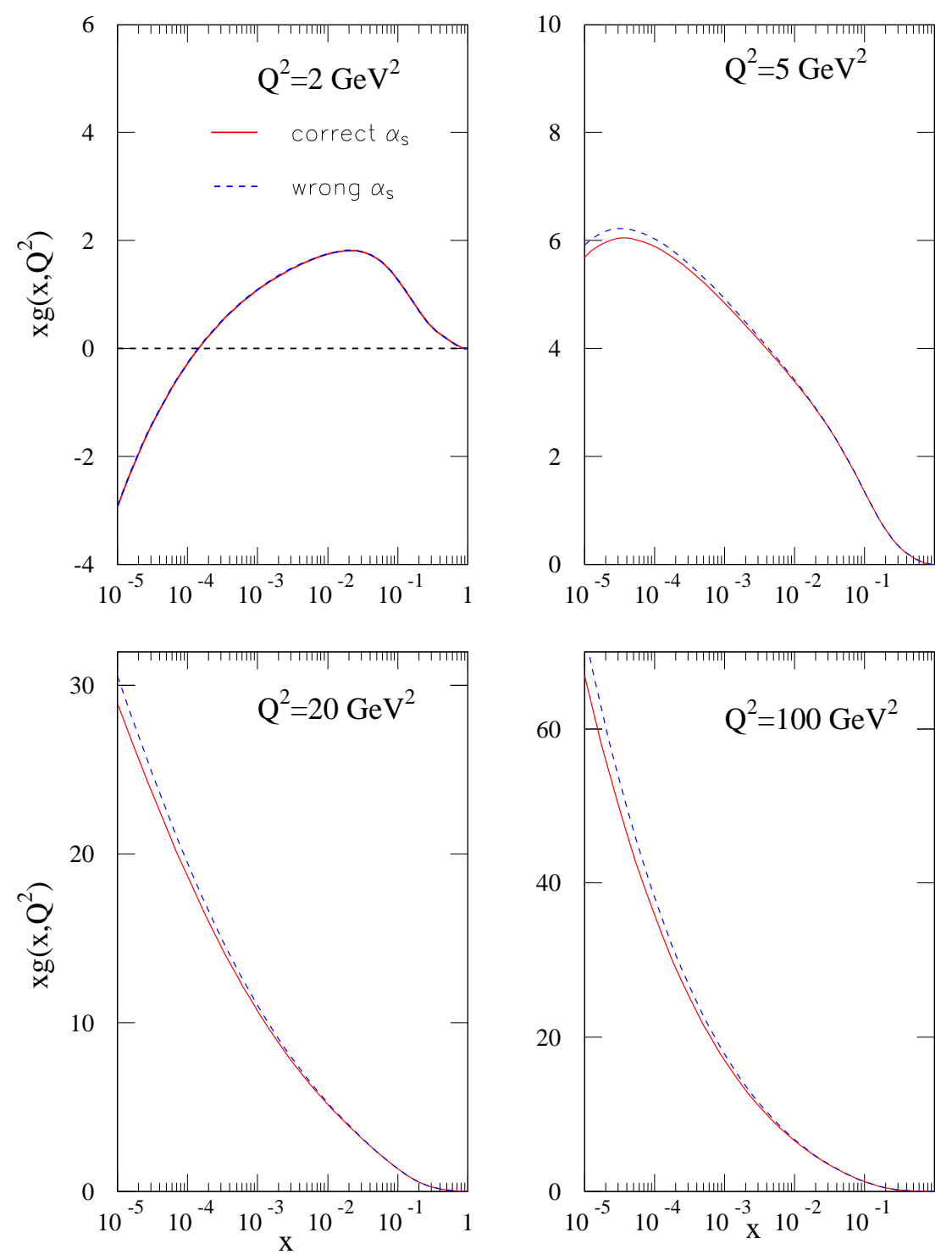

Figure 1: The effect on the gluon distribution of, using in the 3-flavour FF evolution, a different number of active quark flavours in the coupling and the structure functions. The wrong $\alpha_{S}$ corresponds to increasing $n_{f}$ in the coupling at each heavy quark threshold. 
and therefore at this order

$$
\frac{\partial F_{L}}{\partial \ln Q^{2}}=-\beta_{0} \alpha_{S}^{2} C_{L g}^{1} \otimes g+\alpha_{S}^{2} C_{L g}^{1} \otimes P_{g g}^{(0)} \otimes g \quad+\text { quark term. }
$$

Here $\beta_{0}=\left(11-\frac{2}{3} n_{f}\right) / 4 \pi$, whereas $P_{g g}^{(0)}$ contains a term $-\left(\frac{2}{3} n_{f} / 4 \pi\right) \delta(1-z)$, i.e. the gluon loses momentum to the quarks it radiates. Hence, for example, in going from the $n_{f}=3$ renormalization scheme to the $n_{f}=4$ renormalization scheme, the change in these two terms cancels out, leaving the physical quantity independent of choice of number of active quarks $n_{f}$. However if the coupling and the structure functions use a different number of active quarks, as is often done, the cancellation does not occur. The effect of this inconsistent choice on the gluon distribution is shown in Fig.1. The gluon evolved using the incorrect coupling evolves more quickly than that with the correct coupling since the coupling is always larger in the former case. By $Q^{2}=100 \mathrm{GeV}^{2}$ the incorrect gluon is about $10 \%$ larger at $x=0.00001$. At $x \sim 0.05$, where there is little change with $Q^{2}$, the gluons are very similar, but at high $x$ the gluon with the incorrect coupling decreases more quickly and at $Q^{2}=100 \mathrm{GeV}^{2}$ is up to $10 \%$ smaller at $x=0.5$. The error on the gluon due to the mistake in the choice of coupling is generally of similar size to the uncertainty due to experimental errors in [8]. As such, this effect is far from insignificant. A similar effect is seen in the quark distributions, i.e. evolution is too quick, and some effects due to internal quark loops are double-counted.

We make the correctly generated 3- and 4-flavour FFNS parton distributions, which we denote by MRST2004FF3 and MRST2004FF4, generally available ${ }^{3}$. We do this at both NLO and also at LO since LO partons are sometimes needed for LO Monte Carlo programmes (see e.g. [9]). The LO partons are the companions to those in [10], i.e. the MRST2001 LO partons, and as in this previous paper we point out that the LO partons are not able to give a genuinely good global fit and should be used with caution. The NLO 3-flavour gluon and singlet-quark distributions are compared to their VFNS counterparts in Fig.2. The gluon distribution is always bigger in the 3-flavour scheme because of the extra radiation of gluons to charm and bottom quarks in the VFNS. This extra positive evolution outweighs the decreased evolution due to the lower 3-flavour coupling at low $x$. At high $x$ both the lower coupling and the lack of radiation to quarks slow the decrease of the 3-flavour gluon relative to that of the VFNS gluon. These two effects are shown in Fig.3. The 3-flavour gluon is hence always bigger than the VFNS gluon for $Q^{2}>m_{c}^{2}$. Conversely, as seen in Fig.2, the singlet quark distribution is always correspondingly bigger. Almost all of this is difference is due to the presence of the charm and bottom quarks, which carry the extra momentum lost by the VFNS gluon. In order to highlight the difference between the 3-flavour and VFNS gluon we also plot in Fig.4 the ratio of the corresponding $g g$ luminosity functions $\left(\partial \mathcal{L}^{g g} / \partial \tau, \tau=M_{X}^{2} / s\right)$ for producing a heavy particle of mass $M_{X}$ at the LHC. The larger 3-flavour gluon always leads to a larger luminosity, particularly when probing very high $x$ where the gluons differ most. This highlights the extreme care which must be taken in using the correctly defined gluon together with the

\footnotetext{
${ }^{3}$ These parton sets can be found at http://durpdg.dur.ac.uk/hepdata/mrs.html.
} 
3-Flavour Gluon and Singlet against 5-Flavour Gluon and Singlet
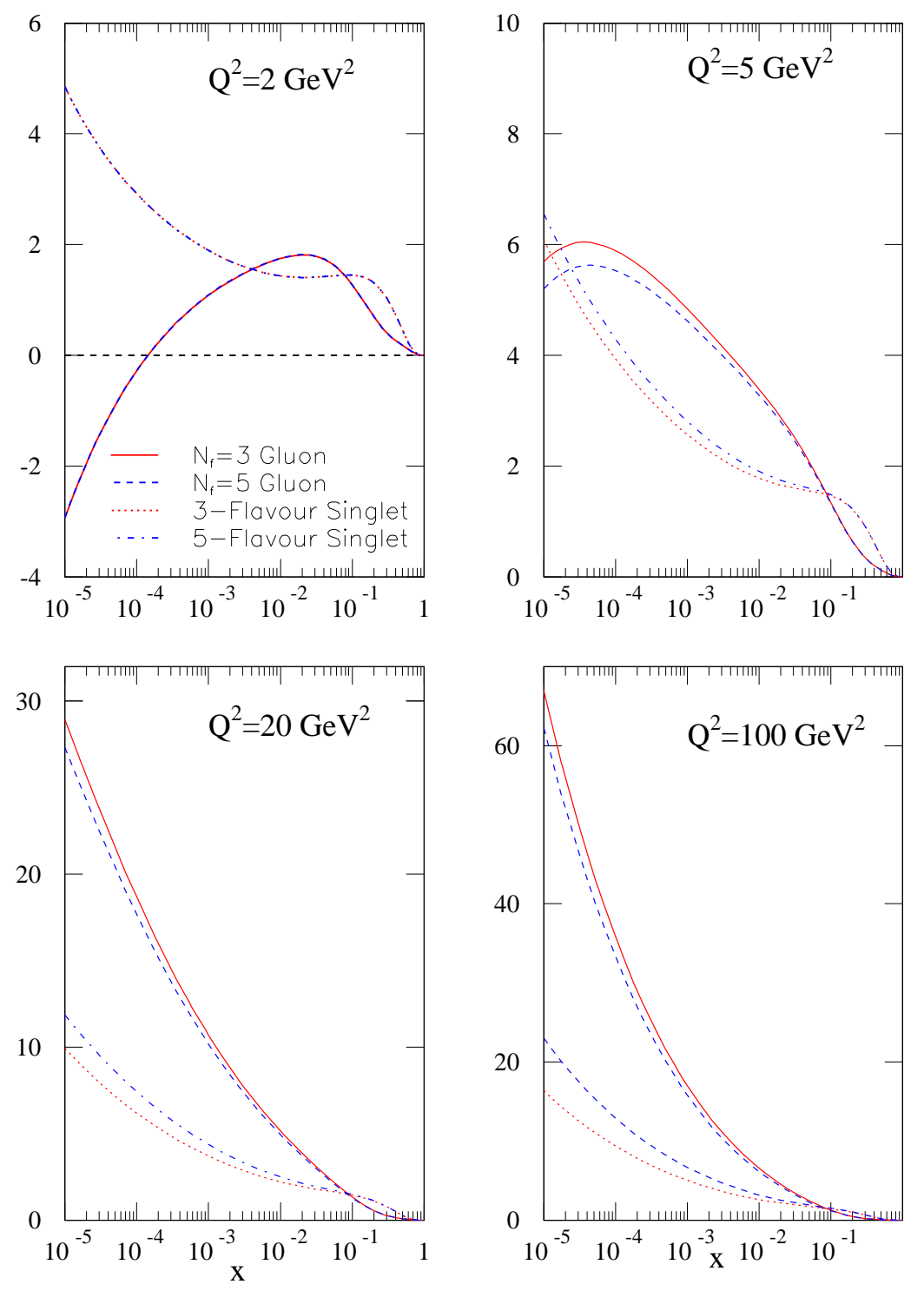

Figure 2: The comparison of the gluon distributions (and the total singlet-quark distributions) in the 3-flavour FFNS and the VFNS. 
correctly defined coupling and hard cross-section in any predictions for particle production at the LHC or Tevatron.

This latter point also illustrates the difficulty in performing a correct global fit to parton distributions with a FFNS, i.e. we need exact versions of the NLO hard cross-sections which should be used with the FFNS partons. All will have logarithms in $Q^{2} / m_{H}^{2}$, which compensate for the missing heavy quark parton distributions, along with other mass effects. (Here the subscript $H=c$ or $b$.) However, these are not known for all processes. Hence, our decision to generate instead sets of FFNS partons which are consistent with our standard partons below $Q^{2}=m_{c}^{2}$. We are easily able to calculate the structure function $F_{2}\left(x, Q^{2}\right)$ in the FFNS scheme however. Note that in this scheme the coefficient functions for heavy flavour production begin at order $\alpha_{S}$, so LO corresponds to $\mathcal{O}\left(\alpha_{S}\right)$ coefficient functions, NLO to $\mathcal{O}\left(\alpha_{S}^{2}\right), \mathcal{O}\left(\alpha_{S}^{3}\right)$, etc.. This is consistent since the $\mathcal{O}\left(\alpha_{S}\right)$ coefficient function contains information on the LO splitting function $P_{q g}^{(0)}$ the $\mathcal{O}\left(\alpha_{S}^{2}\right)$ coefficient function on the NLO splitting function $P_{q g}^{(1)}$, etc., so at each order the evolution of the heavy flavour structure function is roughly correct. The NLO cross-sections for the heavy flavour contributions have long existed as semi-analytic code [11] where the dominant contributions for $W^{2} \rightarrow \infty$ and $W^{2} \rightarrow 4 m_{H}^{2}$ (from above) are analytic and the rest numerical. The NNLO coefficient functions for heavy flavour production are not known yet, though approximate expressions for low $Q^{2}$ are derived in [12], which enables one to define a NNLO VFNS. As another by-product of the definition of the scheme in [12] there exist faster analytic expressions for the NLO coefficient functions which are exact for $Q^{2} / m_{H}^{2} \rightarrow \infty$ and in some cases for $W^{2} \rightarrow 4 m_{H}^{2}$, and the $\left(m_{H}^{2} / Q^{2}\right)$ remainders are provided by fitting the values to analytic functions with a number of free parameters. These final expressions are slightly approximate, but the error in $F_{2}^{H}\left(x, Q^{2}\right)$ is only $\sim 1 \%$, even in the most extreme cases. ${ }^{4}$

The results for the charm structure function and bottom structure function in the FFNS compared to that from the MRST2004 partons using VFNS coefficient functions (using the scheme of [13] appropriate for these partons) are shown in Fig.5, where the factorization scale $\mu^{2}=Q^{2}$ has been used in both cases. Clearly heavy flavour production is generally suppressed in the FFNS compared to the VFNS. ${ }^{5}$ However, it is interesting to note that at extremely high $Q^{2}$ the charm structure function in the VFNS for very small $x$ has come back towards that in the FFNS and even goes a little below the FFNS result. This is because for these very large values of $Q^{2} / m_{c}^{2}$ the extra powers of $\ln Q^{2} / m_{c}^{2}$ resummed by the VFNS procedure are accompanied by multiple convolutions of splitting functions containing information on the loss of charm quarks due to radiation to gluons and even smaller $x$ quarks. This information is missing in the FFNS which only contains the first two powers of $\ln Q^{2} / m_{c}^{2}$ and the accompanying splitting functions.

This general suppression of heavy flavour in FFNS means the quality of the match to the data using the MRST FFNS partons is not expected to be very good in regions where the charm

\footnotetext{
${ }^{4}$ This code can also be found at http://durpdg.dur.ac.uk/hepdata/mrs.html.

${ }^{5}$ This trend has been observed when comparing to recent heavy flavour data using a wide variety of partons and scales [14]. In previous studies the incorrect use of the VFNS coupling in fixed-flavour number schemes has often somewhat masked the suppression.
} 
Evolution of NLO Gluon in FFNS and VFNS
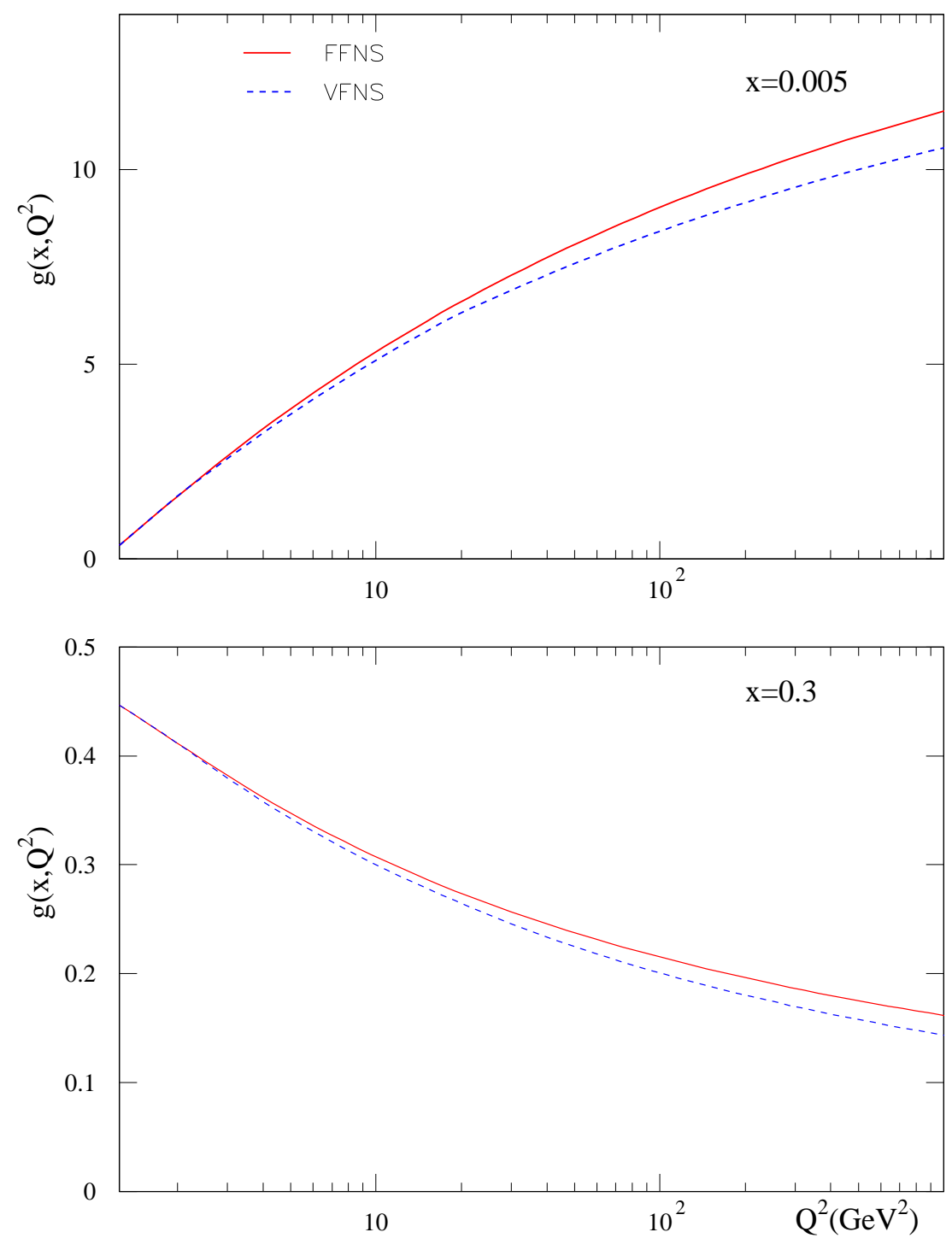

Figure 3: The evolution of the gluon distribution in the 3-flavour FFNS and the VFNS, for typical low and high values of $x$. 


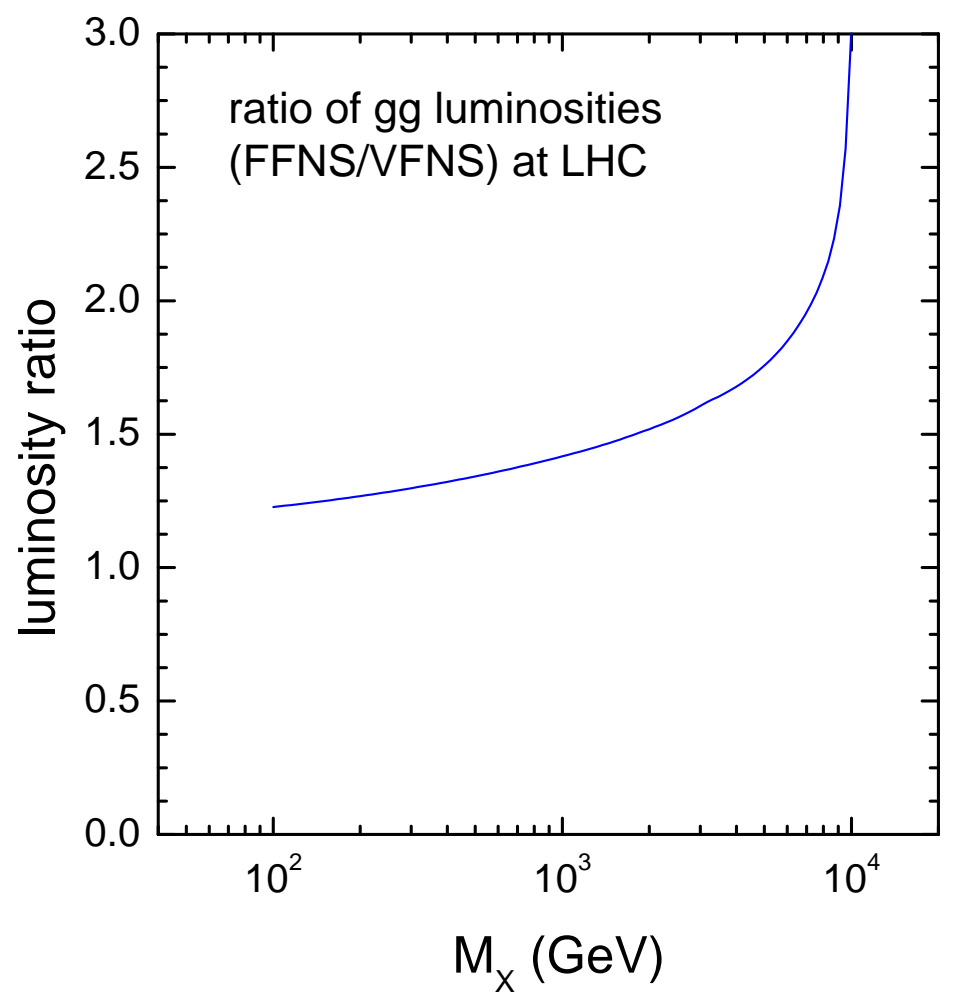

Figure 4: The effect on the luminosity distribution for $g g \rightarrow X$ of using FFNS or VFNS partons. 
Table 1: Quality of the FFNS comparison to some data sets, together with the corresponding $\chi^{2}$ values for the standard NLO VFNS fit to the same data. Here the FFNS partons are not obtained by fitting to data, but rather are generated by a $\mathrm{FF}\left(n_{f}=3\right)$ evolution from the VFNS input distributions.

\begin{tabular}{|lccc|}
\hline Data set & $\begin{array}{c}\text { No. of } \\
\text { data pts }\end{array}$ & VFNS & FFNS \\
\hline H1 $e p$ & 417 & 427 & 1117 \\
ZEUS $e p$ & 356 & 279 & 893 \\
BCDMS $\mu p$ & 179 & 190 & 174 \\
BCDMS $\mu d$ & 155 & 216 & 242 \\
NMC $\mu p$ & 126 & 136 & 164 \\
NMC $\mu d$ & 126 & 103 & 128 \\
SLAC $e p$ & 53 & 50 & 74 \\
SLAC $e d$ & 54 & 56 & 58 \\
E665 $\mu p$ & 53 & 50 & 49 \\
E665 $\mu d$ & 53 & 61 & 63 \\
\hline
\end{tabular}

contribution to the structure function is important. In the Table we show the contributions to $\chi^{2}$ from various subsets of the data fitted in the global analysis which yields the NLO MRST2004 (VFNS) partons [4]. The Table compares these values with the $\chi^{2}$ obtained from the FFNS partons generated using the MRST2004 partons as input at $Q_{0}^{2}=1 \mathrm{GeV}^{2}$. We emphasise that the latter partons are not obtained in a new fit to the data. We see that the $\chi^{2}$ for the HERA (H1, ZEUS) data is indeed significantly larger using the FFNS partons. This is due to the fact that at small $x,\left(\lesssim 10^{-2}\right), F_{2}^{c}$ increases with $Q^{2}$ up to $30 \%$ more slowly in the FFNS. Since $F_{2}^{c}$ is a major component of the total $F_{2}$ at small $x$, this leads to a general undershooting of these HERA data. Note that the description of the fixed-target (BCDMS, SLAC) structure function data is not changed significantly in going from VFNS to FFNS, while the NMC data is slightly sensitive to the charm structure function and is affected a little more. This consistency in the fixed-target data is because the slowing down of the evolution above $Q^{2} \sim m_{c}^{2}$, due to the reduced coupling, is largely compensated by the $n_{f}$ dependence of the NLO splitting functions. If we were to use the VFNS coupling for the FFNS partons the description of the fixed-target data would be worse. As already noted, the smaller $\alpha_{S}$ for the FFNS partons at high $Q^{2}$ is accompanied by a larger gluon distribution at large $x$ due to the slower evolution. These two effects compensate in the description of the Tevatron inclusive jet data which, as far as one can tell without FFNS jet coefficient functions, is similar for both schemes.

We notice that it is the charm (and bottom) data themselves, and hence the description of the small $x$ structure function data, that are the most sensitive to the choice of the scheme for the heavy flavours, which is, of course, not unexpected. Indeed, if we do attempt a fit to as 
Evolution of $\mathrm{NLO} \mathrm{F}_{2}^{\mathrm{c}}\left(\mathrm{x}, \mathrm{Q}^{2}\right)$ in FFNS and VFNS
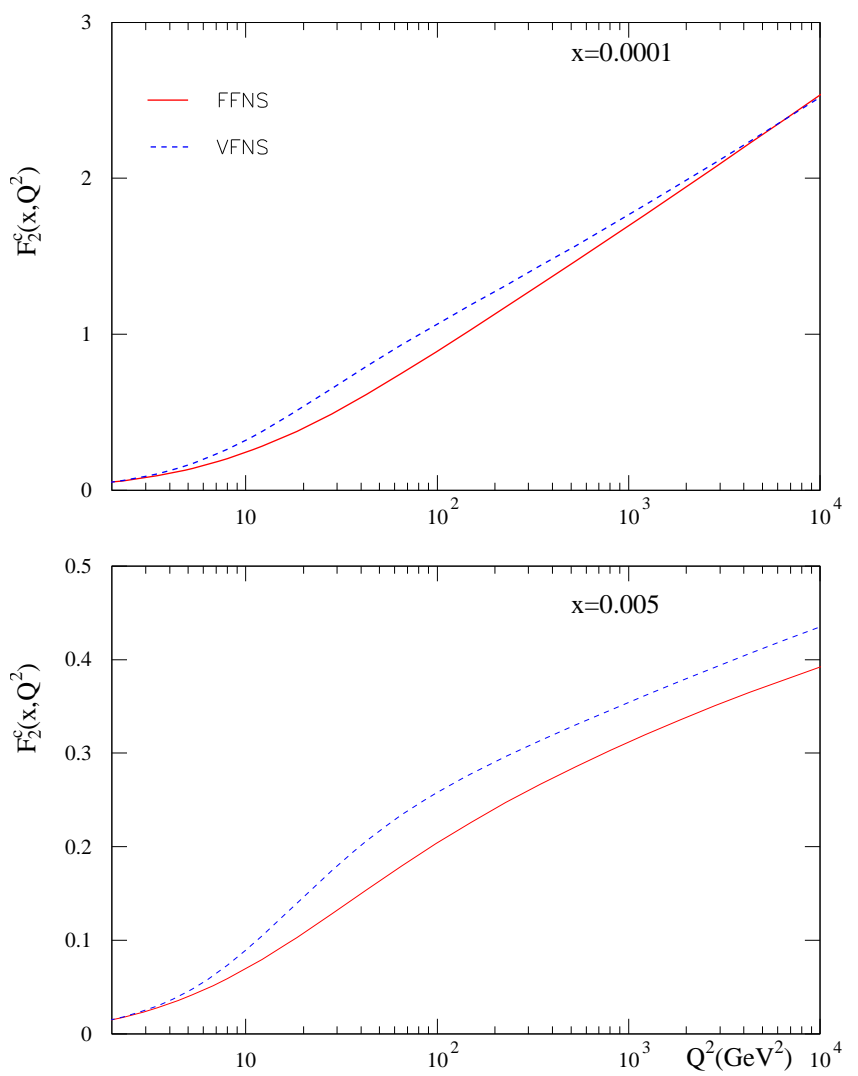

Evolution of $\mathrm{NLO}_{\mathrm{F}}^{\mathrm{b}}\left(\mathrm{x}, \mathrm{Q}^{2}\right)$ in FFNS and VFNS
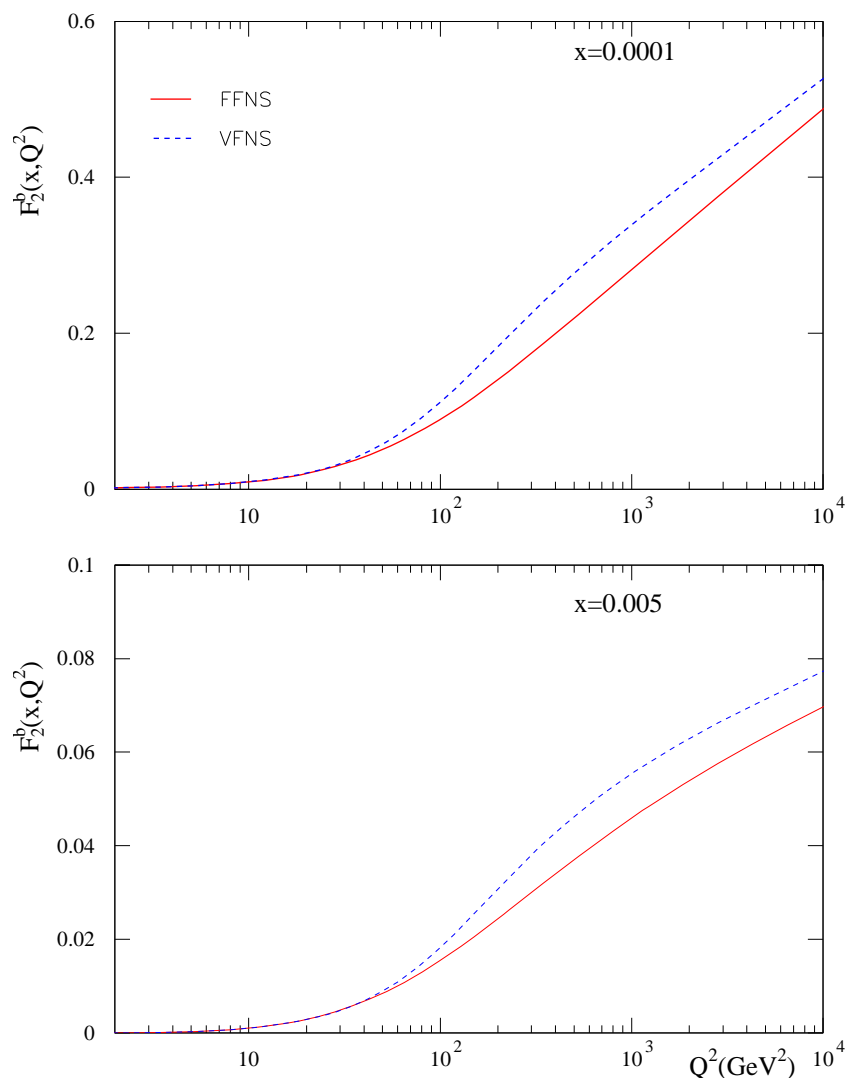

Figure 5: The evolution of $F_{2}^{c}\left(x, Q^{2}\right)$ in the 3-flavour FFNS and VFNS (left). The evolution of $F_{2}^{b}\left(x, Q^{2}\right)$ in the 3 -flavour FFNS and VFNS (right). 
much data in the global fit as possible in the FFNS scheme ${ }^{6}$ our comparison to HERA data is always 80 units of $\chi^{2}$ or so worse than in the VFNS scheme, even though the value of the 3 -flavour coupling increases significantly in an attempt to fit the small- $x$ data more successfully. This increase in the coupling is such that it causes the fit to fixed-target data to deteriorate. We believe that this difficulty in fitting small- $x$ structure function data in a FFNS scheme would be generally observed if the correct definition of the coupling were used and constraints from data sets other than DIS data were applied in such fits. Hence, we conclude that it is always preferable, if possible, to work in a variable-flavour number scheme for parton distributions. However, recognising that this is not always possible for current applications, and also that there is a general interest in FFNS partons, we use this paper to advertise and make available our 3-flavour and 4-flavour scheme partons generated from the same input as our 2004 VFNS partons, but consistently evolved in these two alternative schemes.

\section{Acknowledgements}

RST would like to thank Bryan Webber for discussions on the use of partons in heavy-flavour Monte Carlos and Paul Thompson for asking questions which led to the investigation of the use of the coupling constant in fixed-flavour schemes and for comments on the paper. RST thanks the Royal Society for the award of a University Research Fellowship and ADM thanks the Leverhulme Trust for the award of an Emeritus Fellowship. The IPPP gratefully acknowledges financial support from the UK Particle Physics and Astronomy Research Council.

\section{References}

[1] B.W. Harris and J. Smith, Nucl. Phys. B452 (1995) 109,

B.W. Harris and J. Smith, Phys. Rev. D57 (1998) 2806.

[2] M.L. Mangano, P. Nason and G. Ridolfi, Nucl. Phys. B373 (1992) 295.

[3] S. Frixione, P. Nason and B.R. Webber, JHEP 0308 (2003) 007.

[4] A.D. Martin, R.G. Roberts, W. J. Stirling and R.S. Thorne, Phys. Lett. B604 (2004) 61.

[5] W.J. Marciano, Phys. Rev. D29 (1984) 5801.

[6] CTEQ collaboration, H.L. Lai et al., Eur. Phys. J. C12 (2000) 375.

[7] E. Laenen, S. Riemersma, J. Smith and W.L. van Neerven, Nucl. Phys. B392 (1993) 162.

\footnotetext{
${ }^{6}$ Only the neutral current structure function data may be included completely rigorously. The NLO FFNS coefficient functions are not calculated for charged current structure functions. NLO FFNS coefficient functions are calculated for the Drell-Yan cross-section [15] but not in sufficiently differential form.
} 
[8] A.D. Martin, R.G. Roberts, W.J. Stirling and R.S. Thorne, Eur. Phys. J. C28 (2003) 455.

[9] G. Marchesini and B.R. Webber, Nucl. Phys. B330 (1990) 261.

[10] A.D. Martin, R.G. Roberts, W.J. Stirling and R.S. Thorne, Phys. Lett. B531 (2002) 216.

[11] S. Riemersma, J. Smith and W.L. van Neerven, Phys. Lett. B347 143, (1995).

[12] R.S. Thorne, hep-ph/0601245, to be published in Phys. Rev. D.

[13] R. S. Thorne and R. G. Roberts, Phys. Lett. B421 (1998) 303; Phys. Rev. D57 (1998) 6871.

[14] P. Thompson, private communication.

[15] P.J. Rijken and W.L. van Neerven, Phys. Rev. D52 (1995) 149. 